\title{
UNCOVERING STELLAR ATMOSPHERES WITH GRAVITATIONAL MICROLENSING TELESCOPES
}

\author{
M. DOMINIK \\ University of St Andrews, School of Physics \& Astronomy, \\ North Haugh, St Andrews, KY16 9SS, United Kingdom \\ E-mail: md35@st-andrews.ac.uk
}

\begin{abstract}
A strong differential magnification over the face of the source star passing the caustic created by a binary lens star allows to measure its radial intensity profile with an angular resolution of 20-60 nas from broad-band photometric observations for $\sim 15$ stars per year and to study its chemical composition from time-resolved high-resolution spectroscopy for $\sim 2$ stars per year.
\end{abstract}

\section{Broad-band photometry and limb darkening}

During a period of a few hours to a few days, over which a background source star crosses the caustic due to the gravitational field of a foreground stellar binary passing close to the line-of-sight, it encounters a strong differential magnification over its face, allowing a measurement of its radial intensity profile from the dense and precise sampling of the microlensing lightcurve which shows a characteristic shape $\frac{89}{8}$ With a typical proper motion between lens and source star of $10-30 \mu \mathrm{as}^{-1}$, a sampling interval of $3 \mathrm{~min}$ results in an effective angular resolution of 20-60 nas for the natural gravitational telescope. Using a normalized stellar intensity profile $I_{\lambda}(\rho)$ corresponding to a square-root limb-darkening law, parametrized as

$$
I_{\lambda}(\rho)=\left(1-c_{\lambda} / 3-d_{\lambda} / 5\right)^{-1}\left[1+c_{\lambda}\left(\sqrt{1-\rho^{2}}-1\right)+d_{\lambda}\left(\sqrt[4]{1-\rho^{2}}-1\right)\right],
$$

where $\rho$ denotes the fractional radius, PLANET has published measurements of limb-darkening coefficients for three K-giants and one G/K-subgiant in the galactic bulge, 2346 and together with OGLE, MACHO, MPS, and MOA, one A-dwarf in the SMC 1 Table 1 lists the relevant parameters of these events, while Fig. 1 shows the photometric $I$-band data obtained for the event OGLE 1999-Bulge-23, corresponding to the most precise limb-darkening measurement of $\sim 6 \%$. The capabilities of current microlensing campaigns allow to determine up to 15 limb-darkening coefficients on various types of source stars per year.

\section{Time-resolved spectroscpy and chemical composition}

According to the variation of the abundance of a chemical element with stellar radius, the associated spectral lines vary during a caustic crossing which can be ob- 


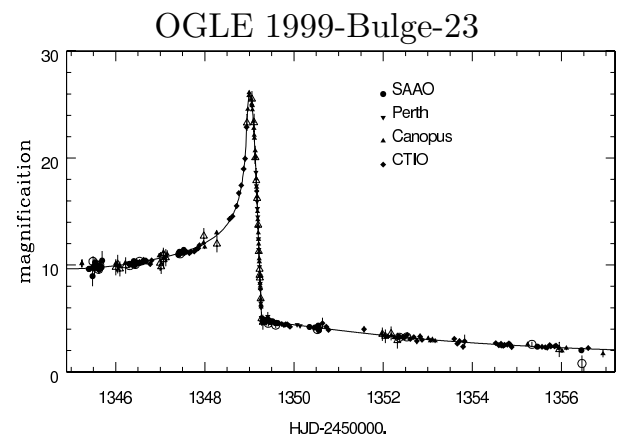

Figure 1. PLANET $I$-band data from four sites during the OGLE 1999-Bulge-23 caustic exit.

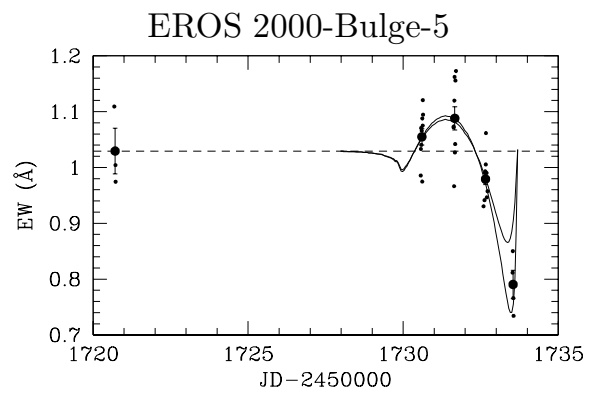

Figure 2. Equivalence-width variation of $\mathrm{H}_{\alpha}$ during the caustic exit of event EROS 2000-Bulge-5 from PLANET observations using the FORS1 spectrograph at the VLT. The big circles with the attached error bars denote nightly averages.

served by high-resolution spectroscopy providing a deep probe of the chemical composition of the source star. 7 Using the FORS1 and UVES spectrographs at the VLT, PLANET has obtained spectra during caustic crossings for two microlensing events and observed a significant variation of the equivalent-width in the $\mathrm{H}_{\alpha}$-line shown in Fig. $[2]^{[5]}$ With allocated UVES target-of-opportunity time, PLANET intends to obtain time-resolved spectra during caustic crossings on 1-2 suitable microlensing events per year.

\section{References}

1. C. Afonso et al., Astrophys. J. 532, 340 (2000).

2. M.D. Albrow et al., Astrophys. J. 522, 1011 (1999).

3. M.D. Albrow et al., Astrophys. J. 534, 894 (2000).

4. M.D. Albrow et al., Astrophys. J. 549, 759 (2001).

5. M.D. Albrow et al., Astrophys. J. 550, L173 (2001).

6. D.L. Fields et al., Astrophys. J. 596, 1305 (2003).

7. B.S. Gaudi and A. Gould, Astrophys. J. 513, 619 (1999).

8. S.H. Rhie and D.P. Bennett, Line Caustic Microlensing and Limb Darkening, preprint astro-ph/9912050 (1999).

9. P. Schneider and R.V. Wagoner, Astrophys. J. 314, 154 (1987). 
Table 1. Limb-darkening measurements by PLANET.

\begin{tabular}{|c|c|c|c|c|c|c|c|}
\hline & & \multirow{2}{*}{$\begin{array}{c}\text { MACHO } \\
\text { 1997-Bulge-28 }\end{array}$} & \multirow{2}{*}{$\begin{array}{c}\text { MACHO } \\
\text { 1997-Bulge-41 }\end{array}$} & \multicolumn{2}{|c|}{$\begin{array}{l}\text { MACHO } \\
\text { 1998-SMC-1 }\end{array}$} & \multirow{2}{*}{$\begin{array}{c}\text { OGLE } \\
\text { 1999-Bulge-23 }\end{array}$} & \multirow{2}{*}{$\begin{array}{c}\text { EROS } \\
\text { 2000-Bulge-5 }\end{array}$} \\
\hline & & & & close binary & wide binary & & \\
\hline \multirow{6}{*}{$\begin{array}{l}\text { type of source star } \\
\text { angular radius } \theta_{\star} \\
\text { stellar radius } R_{\star} \\
\text { source distance } D_{\mathrm{S}} \\
\text { proper motion } \mu\end{array}$} & & K0...K5 III & K III & \multicolumn{2}{|c|}{$\mathrm{A} 5 \ldots \mathrm{A} 7 \mathrm{~V}$} & G7...K2 IV & $\mathrm{K} 2 \ldots \mathrm{K} 4 \mathrm{III}$ \\
\hline & {$[\mu \mathrm{as}]$} & $8 \pm 2$ & $5.56 \pm 0.54$ & $0.082 \pm 0.005$ & $0.089 \pm 0.005$ & $1.86 \pm 0.13$ & $6.62 \pm 0.58$ \\
\hline & {$\left[R_{\odot}\right]$} & $15 \pm 2$ & $10 \pm 2$ & $1.1 \pm 0.1$ & $1.1 \pm 0.1$ & $3.4 \pm 0.6$ & $12 \pm 3$ \\
\hline & {$[\mathrm{kpc}]$} & $8.5 \pm 1.5$ & $8.5 \pm 1.5$ & \multicolumn{2}{|c|}{$60 \pm 2$} & $8.5 \pm 1.5$ & $8.5 \pm 1.5$ \\
\hline & {$\left[\mathrm{km} \mathrm{s}^{-1} \mathrm{kpc}^{-1}\right]$} & $19.4 \pm 2.6$ & $50 \pm 5$ & $1.30 \pm 0.08$ & $1.48 \pm 0.09$ & $22.8 \pm 1.5$ & $31.1 \pm 2.9$ \\
\hline & {$\left[\mu\right.$ as d $\left.^{-1}\right]$} & $11.2 \pm 1.5$ & $29 \pm 3$ & $0.75 \pm 0.05$ & $0.85 \pm 0.05$ & $13.2 \pm 0.9$ & $18.0 \pm 1.7$ \\
\hline duration entry $2\left(t_{\star}^{\perp}\right)_{1}$ & {$[\mathrm{~h}]$} & 37.4 & 11.8 & 6.4 & 6.6 & 8.8 & 26 \\
\hline duration exit $2\left(t_{\star}^{\perp}\right)_{2}$ & {$[\mathrm{~h}]$} & 39.2 & 9.2 & 8.562 & 8.582 & $8.194 \pm 0.072$ & 86 \\
\hline time between c.c.s $T_{12}$ & & $5.08 \mathrm{~h}$ & $4.62 \mathrm{~h}$ & \multicolumn{2}{|c|}{$13.2 \mathrm{~d}$} & $7.3 \mathrm{~d}$ & $27 \mathrm{~d}$ \\
\hline time resolution $\Delta t$ & {$[\mathrm{~min}]$} & 3 & 10 & \multicolumn{2}{|c|}{6} & 5 & 15 \\
\hline angular resolution $\Delta \theta$ & [nas] & 23 & 220 & 3 & 3 & 50 & 180 \\
\hline 1.d. coefficients $\quad c_{\mathrm{I}}$ & & $0.40 \pm 0.08$ & $0.52 \pm 0.10$ & $0.24 \pm 0.05(\mathrm{SAAO})$ & $0.21 \pm 0.05(\mathrm{SAAO})$ & $0.632_{-0.037}^{+0.047}$ & $0.552 \pm 0.090$ \\
\hline 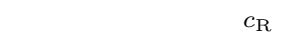 & & - & - & $0.24 \pm 0.05$ (EROS) & $0.21 \pm 0.05$ (EROS) & -0.037 & - \\
\hline & & - & - & $0.06 \pm 0.33(\mathrm{CTIO})$ & $0.06 \pm 0.36($ CTIO $)$ & - & - \\
\hline$c_{\mathrm{B}}$ & & - & - & $0.42 \pm 0.04(\mathrm{EROS})$ & $0.40 \pm 0.04($ EROS $)$ & - & - \\
\hline$c_{\mathrm{V}}$ & & $0.55 \pm 0.11$ & - & $0.55 \pm 0.11(\mathrm{SAAO})$ & $0.50 \pm 0.11(\mathrm{SAAO})$ & $0.786_{-0.080}^{+0.080}$ & - \\
\hline$d_{\mathrm{I}}$ & & $0.37 \pm 0.07$ & - & - & - & -0.078 & $0.01 \pm 0.14$ \\
\hline$d_{\mathrm{V}}$ & & $0.44 \pm 0.09$ & - & - & - & - & - \\
\hline
\end{tabular}

